\title{
Time-dependent rotational stability of dynamic planets with elastic lithospheres
}

\section{Citation}

Chan, N.-H., J. X. Mitrovica, A. Daradich, J. R. Creveling, I. Matsuyama, and S. Stanley. 2014. "Time-Dependent Rotational Stability of Dynamic Planets with Elastic Lithospheres." Journal of Geophysical Research: Planets 119 (1): 169-88. https://doi.org/10.1002/2013je004466.

\section{Permanent link}

http://nrs.harvard.edu/urn-3:HUL.InstRepos:41401427

\section{Terms of Use}

This article was downloaded from Harvard University's DASH repository, and is made available under the terms and conditions applicable to Other Posted Material, as set forth at http:// nrs.harvard.edu/urn-3:HUL.InstRepos:dash.current.terms-of-use\#LAA

\section{Share Your Story}

The Harvard community has made this article openly available.

Please share how this access benefits you. Submit a story.

Accessibility 


\section{Journal of Geophysical Research: Planets}

\author{
RESEARCH ARTICLE \\ 10.1002/2013JE004466 \\ Key Points: \\ - We extend a non-linear, \\ time-dependent theory of true polar \\ wander (TPW) \\ - Our extended theory includes \\ stabilization by the so-called \\ "remnant bulge" \\ -We illustrate the extended theory \\ using models of TPW on both Earth \\ and Mars
}

\section{Correspondence to:}

N.-H. Chan,

nhchan@fas.harvard.edu

\section{Citation:}

Chan, N.-H., J. X. Mitrovica, A. Daradich, J. R. Creveling, I. Matsuyama, and S. Stanley (2014), Time-dependent rotational stability of dynamic planets with elastic lithospheres, J. Geophys. Res. Planets, 119, 169-188, doi:10.1002/2013JE004466.

\section{Received 18 JUN 2013}

Accepted 14 DEC 2013

Accepted article online 16 DEC 2013

Published online 28 JAN 2014

\section{Time-dependent rotational stability of dynamic planets with elastic lithospheres}

\author{
N.-H. Chan' ${ }^{\text {, J. X. Mitrovica }{ }^{1} \text {, A. Daradich' }{ }^{2} \text {, J. R. Creveling }}{ }^{3}$, I. Matsuyama ${ }^{4}$, and S. Stanley ${ }^{5}$ \\ 'Department of Earth and Planetary Sciences, Harvard University, Cambridge, Massachusetts, USA, ${ }^{2}$ Department of Earth \\ Sciences, University of Ottawa, Ottawa, Ontario, Canada, ${ }^{3}$ Department of Geological and Planetary Sciences, California \\ Institute of Technology, Pasadena, California, USA, ${ }^{4}$ Lunar and Planetary Laboratory, University of Arizona, Tucson, Arizona, \\ USA, ${ }^{5}$ Department of Physics, University of Toronto, Toronto, Ontario, Canada
}

\section{Introduction}

Redistributions of mass on a planet, whether driven by internal processes such as thermochemical convection, external processes such as the growth and ablation of ice sheets, or some combination of the two (e.g., volcanism), will drive reorientations of the rotation axis relative to the surface geography over a broad spectrum of timescales. Long-term, secular components of this reorientation are known as true polar wander (henceforth, TPW).

The analysis of the rotational stability of terrestrial planets, and in particular TPW, is a classic problem that dates, in the geophysical literature, to the canonical midtwentieth century study of Gold [1955]. Gold's conceptual model of load-induced TPW is illustrated schematically in Figure 1a. We begin with a rotating planet whose gravitational figure has fully relaxed to an imposed centrifugal potential, i.e., a planet with a hydrostatic figure (Figure 1a1). If the planet is subject to a positive load, then the load will be thrown outward toward the equator, or, for an observer fixed to the planet, the pole will migrate (or wander) away from the load (Figure 1a2). This wander will be opposed by the rotational bulge, since it represents an excess mass that will resist displacement off the equator. However, Gold [1955] argued that this resistance is transient, since the Earth will viscously deform in response to the perturbed centrifugal potential so as to bring the bulge back onto the equator (Figure 1a3). Once this adjustment takes place, the load is free to move farther away from the rotation axis and the process will continue in incremental steps (Figure 1a4) until the load has reached the equator (Figure 1a5). In the conceptual model of Gold [1955], planetary rotation is inherently unstable because a load of any size will ultimately migrate to the equator.

We can extend these ideas to the hypothetical case of a planet with an elastic lithosphere, the initial figure of which is not hydrostatic (Figure 1b1). This case is consistent with a nonrotating, spherical planet (Figure 1b0) with an elastic lithosphere that is spun up to a final form in which all viscous stresses have relaxed. As in Figure 1a2, loading this planet will drive TPW, and this polar motion will meet transient resistance from a 

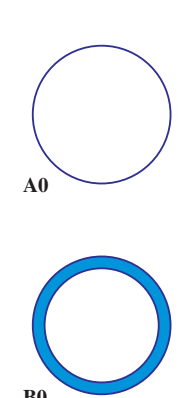

B0

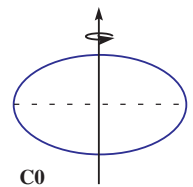

C0

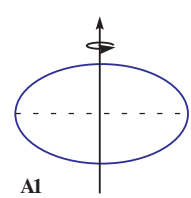

A1

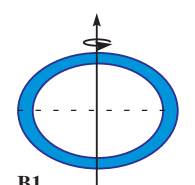

B1

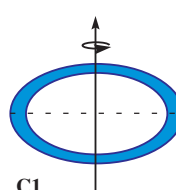

C1

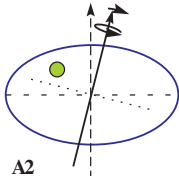

A2

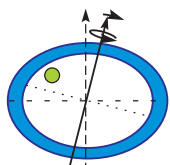

B2

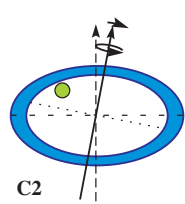

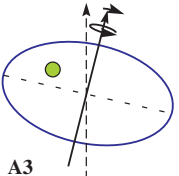

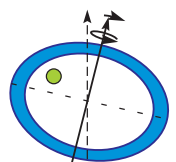

B3

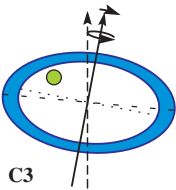

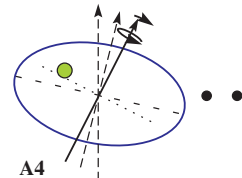

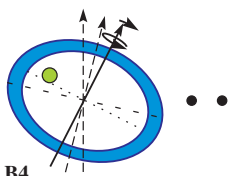

B4

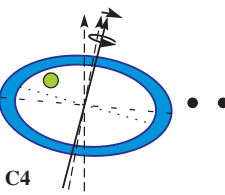

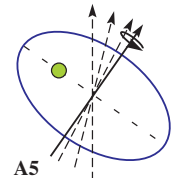

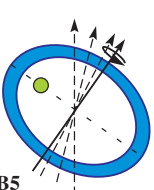

B5

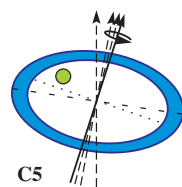

Figure 1. Schematic illustration of the physics of TPW. The green disk represents an internal load, the solid-blue outer shell is an elastic lithosphere, the solid arrow (with spin and TPW directions specified near the tip) is the rotation vector, and the long-dashed arrows are previous rotation vectors within the same series. On each frame, the short-dashed line within the interior of the model planet denotes the plane of the rotational bulge, while the dotted line is the rotational equator (i.e., $90^{\circ}$ from the contemporaneous rotation axis). (a0-b5) The cases where the rotational bulge can ultimately relax perfectly to any change in the orientation of the rotation axis (whenever this occurs, the axis of the bulge and the equator, denoted by the short-dashed and dotted lines, respectively, will coincide). In this case, which is the scenario discussed by Gold [1955], an equilibrium theory predicts that TPW will ultimately reorient the load to the equator (Figures $1 \mathrm{a} 5$ or $1 \mathrm{~b} 5$ ). ( $(\mathrm{c} 0-\mathrm{c5}$ ) The case where the initial state is hydrostatic, but where any readjustment of the bulge will introduce elastic stresses in the (initially unstressed) lithosphere. In this case, the rotational bulge cannot adjust perfectly to a change in the orientation of the rotation axis, and the final position of the load will not be the equator (Figure 1c5). This final state is predicted by the equilibrium theory of Matsuyama et al. [2006] (see equation (28)) and it is governed by a balance between the load-induced forcing and the resistance provided by the elastic lithosphere (the latter stabilization is termed the "remnant bulge"; see also Willemann [1984]). The schematics in the figure are shown in a reference frame fixed to the location on the load. While the figure presents the case of an internal loading, the same physics applies to external loads; however, in this case the no-lithosphere scenario shown in Figure 1a must assume that the surface mass load is never fully compensated.

rotational bulge that will ultimately reorient perfectly to any new rotational state (Figure $1 \mathrm{~b} 3$ ). The process will continue and, once again, the load will reach the equator (Figure 1b5). The key point in Figures 1a and $1 \mathrm{~b}$ is that the rotation axis is inherently unstable whenever the rotational bulge can relax so that the initial form of the planet is reestablished around the new rotational state, whether this initial form is hydrostatic or not. In this case, in the terminology of Gold [1955], the rotating planet is said to lose all memory of previous rotational states.

Goldreich and Toomre [1969] provided a theoretical framework for the conceptual model of Gold [1955] and considered rotational stability in the case of multiple loads. They demonstrated, for slow changes in the shape of a quasi-rigid body, that the angle between the angular momentum vector and the principal axes is an adiabatic invariant. In the context of TPW, this invariance means that the rotation axis will remain aligned with the principal axis of inertia whenever changes in the shape of the planet are slow.

It is important to note that Gold's conceptual model predicts the final orientation of the rotation axis (i.e., Figures $1 \mathrm{a} 5$ and $1 \mathrm{~b} 5$ ), but not the timescale of TPW (i.e., Figures 1a1-1a4 and 1b1-1b4). A variety of methodologies have been developed within the geophysical literature to estimate the time dependence of TPW driven by mantle convection, including both nonlinear [Ricard et al., 1993; Steinberger and O'Connell, 2002; Tsai and Stevenson, 2007] and linearized [Chan et al., 2011; Cambiotti et al., 2011] treatments of the governing equations. Ricard et al. [1993] made use of the adiabatic invariance described by Goldreich and Toomre [1969] and incorporated perturbations in the moment of inertia tensor associated with: (i) time-dependent mass redistribution, including boundary deformations, associated with internal convective forcing, and (ii) the time-dependent, viscous response of the rotational bulge to the perturbed centrifugal potential. They expressed the latter contribution in terms of a long timescale, asymptotic expansion of the viscoelastic tidal Love number at spherical harmonic degree 2.

The conceptual model of Gold [1955] was extended by Willemann [1984] [see also Matsuyama et al., 2006] to incorporate stabilization associated with an elastic lithosphere, as illustrated in Figure 1c. Consider an initially hydrostatic planet characterized by an unstressed elastic lithosphere (Figure 1c1). This situation may arise in several ways. First, an elastic lithosphere may gradually cool out of a protoplanet that, at least 
initially, had no lithosphere. In this case, the creation of an unstressed elastic lid would not alter the gravitational figure of the planet (i.e., the evolution from Figure 1c0 to Figure $1 \mathrm{c} 1$ ). Alternatively, one can imagine a planet with a rotation vector that has been stable over a timescale long enough that the viscous stresses in even a high-viscosity lithosphere would have relaxed. The planets in Figures $1 \mathrm{~b} 1$ and $1 \mathrm{c} 1$ have the same rotation rate; the important distinction between them is that the lithosphere in the former is stressed in the preloaded state and, as a consequence, the gravitational figure of the planet is not hydrostatic. As discussed by Willemann [1984] and Matsuyama et al. [2006], the distinction has profound implications for rotational stability [see also Daradich et al., 2008].

Consider a scenario in which the planet in Figure 1c1 is subject to a load. As before, the pole will move away from the load, the rotational bulge will act to resist this motion, and this resistance will gradually weaken as the rotational bulge readjusts to the new orientation of the rotation axis. However, in contrast to the previous scenarios, elastic stresses induced in the lithosphere by TPW will prevent the rotational bulge from adjusting perfectly to the new rotational state. That is, the rotational bulge remains misaligned with the geographic equator in Figure 1c3. Nevertheless, the (imperfect) adjustment of the rotational bulge will permit further TPW and the process will continue until elastic stresses in the lithosphere balance the forcing associated with the load (Figure 1c5). In this case, the final position of the load will not be the equator. The stabilization associated with TPW-induced elastic stresses is known as the remnant bulge [Willemann, 1984] (Figure 1c5).

Willemann [1984] concluded that the remnant bulge stabilization is independent of the elastic thickness of the lithosphere. An extension and minor correction of his derivation by Matsuyama et al. [2006] demonstrated, in contrast, that the magnitude of TPW would be dependent on the lithospheric thickness; however, this dependence is only significant for planets with relatively thin lithospheres. As in Gold [1955] and Goldreich and Toomre [1969], Willemann [1984] and Matsuyama et al. [2006] were concerned with equilibrium theories that predicted the final state of the rotation axis in response to a loading.

Chan et al. [2011] derived a linearized (valid for small-amplitude TPW), time-dependent treatment of convection-driven TPW that incorporated stabilization by both the remnant bulge and the delayed viscous adjustment of the rotational bulge. In this paper, we derive a nonlinear, time-dependent rotational stability theory that incorporates both of these stabilization mechanisms. In our derivation, the first of these stabilization mechanisms is incorporated using the method first outlined by Ricard et al. [1993]. The derivation is valid for both internal and external loading, and we will describe the minor modifications necessary to move from one application to the other. In this regard, one might interpret this manuscript as either an extension of the Ricard et al. [1993] study to incorporate remnant bulge stabilization, or an extension of the equilibrium theory of Matsuyama et al. [2006] to treat time-dependent rotational stability. A preliminary discussion of this extended theory, in which the governing equation was provided without derivation, may be found in Creveling et al. [2012].

In the next section, we begin with a detailed mathematical derivation of the governing equations. Following this, we present a series of illustrative numerical simulations that adopt model parameters consistent with the Earth and Mars. Recent finite element modeling has shown that the Earth's broken lithosphere has an effective elastic thickness more than an order of magnitude smaller than the mean plate thickness when considering its impact on rotational stability [Creveling et al., 2012]. In the simulations described below, we generally adopt a lithospheric thickness of $15 \mathrm{~km}$, which is above the upper bound of $10 \mathrm{~km}$ suggested by Creveling et al. [2012]; however, to illustrate the physics of the stabilization we consider the sensitivity of the results to variations in this parameter over the range of $5-25 \mathrm{~km}$. The scenario in Figure $1 \mathrm{c} 1$ is more directly appropriate for Mars, which has an elastic lithosphere with a thickness of several hundred kilometers, but a form that is very close to hydrostatic [Daradich et al., 2008].

\section{Mathematical Formulation}

\subsection{Conservation of Angular Momentum: Euler Equation}

In a rigid body subject to no external torques, angular momentum is conserved and time variations in the angular velocity vector, $\omega$, are governed by the standard Euler equation,

$$
\frac{\mathrm{d} L}{\mathrm{~d} t}+\omega \times \boldsymbol{L}=0
$$


where $\boldsymbol{L}$ is the angular momentum vector. The first term on the left-hand side of equation (1) represents the change of $L$ as measured by an observer fixed to the rotating body, while the sum of the two terms is the change measured by an observer in inertial space. When the body is deformable, the moment of inertia tensor, $\mathbf{I}$, becomes variable in time, such that $\boldsymbol{L}(t)=\mathbf{I}(t) \cdot \boldsymbol{\omega}(t)$. In this case, if the coordinate system is chosen to coincide with the instantaneous principle axes of $\mathbf{I}(t)$, equation (1) generalizes into the so-called Liouville equation.

In geophysical applications, the broad spectrum of time variability in both $\mathbf{I}$ and $\boldsymbol{\omega}$ makes it challenging, in general, to solve the nonlinear Liouville equation. As a consequence, problems involving timescales much longer than a year have been solved using various approximations. For example, in the case where perturbations to both $\mathbf{I}$ and $\omega$ are relatively small, the Euler equation can be linearized around an initial state. This approach has been the standard methodology in predicting perturbations in Earth rotation driven by ice-age loading, where TPW is of order $1^{\circ}$ or less [Sabadini and Peltier, 1981; Wu and Peltier, 1984; Mitrovica et al., 2005]. In this regard, Mitrovica et al. [2005] demonstrated that traditional ice-age formulations [Sabadini and Peltier, 1981; Wu and Peltier, 1984; Mitrovica and Milne, 1998] introduced significant error in predicting TPW by ignoring stabilization associated with both the remnant bulge (Figure 1c) and long-term (relative to ice-age timescales) excess ellipticity of the Earth's figure.

Chan et al. [2011] extended the linearized Mitrovica et al. [2005] formulation to consider TPW driven by mantle convection; they argued that the error incurred in such predictions is only 10\%-20\% when TPW of $\sim 30^{\circ}-40^{\circ}$ is driven by internal convective forcing concentrated at midlatitudes. The Chan et al. [2011] stability theory incorporates stabilization associated with both the delayed, viscoelastic adjustment of the rotational bulge and the elastic strength in the lithosphere (i.e., the remnant bulge).

A variety of other methodologies have been developed to model convection-driven TPW [Ricard et al., 1993; Steinberger and O'Connell, 2002; Tsai and Stevenson, 2007]. Of particular interest here is the Ricard et al. [1993] approach, which has commonly been adopted within the mantle convection community [e.g., Richards et al., 1999]. Their treatment solves equation (1) under the assumption of very long timescale forcing and includes stabilization due to delayed adjustment of the bulge (but not the remnant bulge); in this (very long timescale) case, the governing equation reduces to an eigenvalue problem [Gold, 1955; Lefftz, 1991; Ricard et al., 1993]:

$$
\mathbf{I} \omega=\lambda \omega
$$

In this case, the evolution of $\omega$ may be calculated by diagonalizing $\mathbf{I}$ at each time step.

In the derivations below, we adopt the approximation inherent to equation (2), and the problem reduces to finding all contributors to the moment of inertia tensor, I. In general, this tensor can be decomposed into three components: (i) the initial (background) equilibrium form of the planet, (ii) any deformations of the planet arising from perturbations in the centrifugal potential, and (iii) mass loads and their associated boundary (surface or internal) deformations.

\subsection{Viscoelastic Love Numbers}

For a spherically symmetric, linear viscoelastic body, the deformation components within the contributions (ii) and (iii) of the last paragraph are commonly described in terms of load and tidal (or tidal effective) viscoelastic $k$ Love numbers at spherical harmonic degree 2 [Peltier, 1974]. In the time domain, these Love numbers may be written, respectively, as

$$
\begin{aligned}
& k^{L}(t, \mathrm{LT})=k^{L, E} \delta(t)+\sum_{k=1}^{K} r_{k}^{\prime} e^{-s} k^{t}, \\
& k^{T}(t, \mathrm{LT})=k^{T, E} \delta(t)+\sum_{k=1}^{K} r_{k}^{\prime \prime} e^{-s} k^{t},
\end{aligned}
$$

where the superscript $E$ denotes the elastic component of the Love numbers, $\delta(t)$ is the Dirac delta function, $r_{k}^{\prime}$ and $r_{k}^{\prime \prime}$ are the amplitudes of a set of $K$ normal modes of viscoelastic decay associated with the load and tidal response, respectively. The $s_{k}$ are the associated characteristic decay times for these modes, which are the same for both the loading and tidal responses. The amplitudes and decay times are a function of the 
internal structure of the planet. For reasons that will become clear below, we have made the dependence of the Love numbers on the elastic thickness of the lithosphere, LT, explicit. In the Laplace transform domain (i.e., s-domain), the Love numbers have the form

$$
\begin{aligned}
& k^{L}(s, \mathrm{LT})=k^{L, E}+\sum_{k=1}^{K} \frac{r_{k}^{\prime}}{s+s_{k}}, \\
& k^{T}(s, \mathrm{LT})=k^{T, E}+\sum_{k=1}^{K} \frac{r_{k}^{\prime \prime}}{s+s_{k}} .
\end{aligned}
$$

Taking the limit as $s \rightarrow 0$, we have the so-called fluid Love numbers,

$$
\begin{aligned}
& k_{f}^{L}(\mathrm{LT})=k^{L, E}+\sum_{k=1}^{K} \frac{r_{k}^{\prime}}{s_{k}}, \\
& k_{f}^{T}(\mathrm{LT})=k^{T, E}+\sum_{k=1}^{K} \frac{r_{k}^{\prime \prime}}{s_{k}} .
\end{aligned}
$$

The fluid Love numbers characterize the response of the planet after all viscous stresses have relaxed; they are dependent on the thickness of the purely elastic lithosphere, but not on viscosity. The hydrostatic response of the planet can be expressed using fluid Love numbers computed with $L T=0$. Following Matsuyama et al. [2006], we will denote these special $(L T=0)$ cases as $k_{f}^{T, *}$ and $k_{f}^{L, *}$, respectively.

In the next subsection, we consider the case where TPW driven by (external or internal) loading is only stabilized by the delayed, viscoelastic adjustment of the rotational bulge, i.e., the cases shown by Figures $1 \mathrm{a}$ and $1 \mathrm{~b}$. Following this, we extend the theoretical formulation to incorporate stabilization associated with the remnant bulge, as in Figure 1c.

\subsection{Transient Stabilization by the Rotational Bulge}

In the initial, unperturbed state, we adopt a coordinate system aligned with the principal axis of inertia, such that the angular velocity vector is given by $\Omega=[0,0, \Omega]$. Making use of viscoelastic Love number theory, a general expression for the time-dependent inertia tensor in the scenarios of Figures $1 \mathrm{a}$ and $1 \mathrm{~b}$ is given by

$$
\begin{aligned}
\mathrm{I}_{i j}(t)= & \mathrm{I}_{0} \delta_{i j}+\frac{a^{5}}{3 G} k_{f}^{T}(\mathrm{LT}) \Omega^{2}\left(\delta_{i 3}-\frac{1}{3}\right) \delta_{i j} \\
& +\frac{a^{5}}{3 G} k^{T}(t, \mathrm{LT}) *\left\{\left[\omega_{i}(t) \omega_{j}(t)-\frac{1}{3} \omega^{2}(t) \delta_{i j}\right]-\Omega^{2}\left(\delta_{i 3}-\frac{1}{3}\right) \delta_{i j}\right\}+\mathrm{I}_{i j}^{L}(t),
\end{aligned}
$$

where $\delta_{i j}$ is the Kronecker delta, $a$ is the radius of the Earth, $G$ is the gravitational constant, $\omega$ is the magnitude of the $\omega$ vector, and the $*$ denotes a time convolution. The first two terms on the right-hand side of the equation represent the inertia tensor in the initial, preloaded state of the planet (Figure 1a1, where LT =0, and Figure $1 \mathrm{~b} 1$, where $L T \neq 0$ ). The first of these terms is the moment of inertia of the spherical, nonrotating planet; the second is the (infinite time) departure from sphericity driven by the centrifugal potential associated with the initial angular velocity vector, $\mathbf{\Omega}$. The third term involves a time convolution between the tidal Love number and the perturbation in the centrifugal potential from this initial state. This time convolution introduces a delayed, viscous response of the rotational bulge to the evolving rotation vector, and this delay acts to stabilize the pole [Ricard et al., 1993]. The last term is the load inertia tensor.

\subsubsection{The Equilibrium Theory of Gold [1955]}

The physics described by Gold [1955] was concerned with the response of the rotating system over timescales much longer than those that govern the viscous adjustment of the rotational bulge. In this limit, the modes of viscoelastic decay associated with the $k^{T}$ Love number will be fully relaxed, and equation (9) simplifies to

$$
\mathrm{I}_{i j}(t)=\mathrm{I}_{0} \delta_{i j}+\frac{a^{5}}{3 G} k_{f}^{T}(\mathrm{LT})\left[\omega_{i}(t) \omega_{j}(t)-\frac{1}{3} \omega^{2}(t) \delta_{i j}\right]+\mathrm{I}_{i j}^{L}(t) .
$$

The first two terms on the right-hand side represent the response of the planet in the fluid limit to the (very slowly) evolving centrifugal potential. This so-called equilibrium form indicates that, under the assumptions used to derive equation (9), the rotational bulge ultimately adjusts perfectly to any changes in the angular 
velocity vector, $\omega$. As discussed above, TPW is computed by applying the expression equation (10) in the eigenvalue equation (2). In this case, the equilibrium form does not contribute, and the time-dependent orientation of the rotation axis (i.e., TPW) is given by the principal axis of the load inertia tensor $\mathrm{I}_{i j}^{L}(t)$ with maximum moment; hence, the load will always reach the equator (Figures $1 \mathrm{a} 5$ and $1 \mathrm{~b} 5$ ). The scenarios shown in Figures $1 \mathrm{a}$ and $1 \mathrm{~b}$ are distinguished by the choice of $\mathrm{LT}$. In the former case, where $\mathrm{LT}=0$, the planet is in hydrostatic equilibrium in both the initial and final states (Figures 1a1 and 1a5).

2.3.2. Ricard et al. [1993]

Whereas Gold [1955] was concerned with the final, equilibrium state of the load, Ricard et al. [1993] was concerned with the timescale of polar motion toward this state; that is, the time-dependent evolution shown schematically in Figures $1 \mathrm{a} 2-1 \mathrm{a} 5$ or $1 \mathrm{~b} 2-1 \mathrm{~b} 5$. This time dependence is governed by equation (9). If we once again collect and cancel the terms in $\Omega$, this equation simplifies to

$$
\mathrm{I}_{i j}(t)=\mathrm{I}_{0} \delta_{i j}+\frac{a^{5}}{3 G} k^{T}(t, \mathrm{LT}) *\left[\omega_{i}(t) \omega_{j}(t)-\frac{1}{3} \omega^{2}(t) \delta_{i j}\right]+\mathrm{I}_{i j}^{L}(t) .
$$

Ricard et al. [1993] used the convolution theorem to express the time convolution in equation (11) as a simple multiplication in the Laplace transform (s) domain. In this context, they derived the following approximation for the $s$-domain $k^{T}$ Love number in the small $s$ limit:

$$
k^{T}(s, \mathrm{LT}) \approx k_{f}^{T}(\mathrm{LT})\left[1-T_{1} s\right],
$$

where

$$
T_{1} \equiv \frac{1}{k_{f}^{T}(\mathrm{LT})} \sum_{k=1}^{K} \frac{r_{k}^{\prime \prime}}{s_{k}^{2}} .
$$

The small-s approximation is consistent with the assumption of a long timescale forcing that permits the application of the eigenvalue approach (equation (2)) for predicting TPW. Using the expression (12) in the s-domain form of equation (11), and inverting the result back into the time domain, yields [Ricard et al., 1993]

$$
\begin{aligned}
\mathrm{I}_{i j}(t)= & \mathrm{I}_{0} \delta_{i j}+\frac{a^{5}}{3 G} k_{f}^{T}(\mathrm{LT})\left[\omega_{i}(t) \omega_{j}(t)-\frac{1}{3} \omega^{2}(t) \delta_{i j}\right] \\
& -\frac{a^{5}}{3 G} k_{f}^{T}(\mathrm{LT}) T_{1}\left[\dot{\omega}_{i}(t) \omega_{j}(t)+\omega_{i}(t) \dot{\omega}_{j}(t)-\frac{2}{3} \omega_{\ell}(t) \dot{\omega}_{\ell}(t) \delta_{i j}\right]+\mathrm{I}_{i j}^{L}(t) .
\end{aligned}
$$

Following equation (2), Ricard et al. [1993] computed TPW by tracking the time-dependent principal axis of the inertia tensor $\mathrm{I}_{i j}(t)$ with maximum moment. As in the last section, the first two terms on the right-hand side of equation (13), which represent the equilibrium form, do not impact the diagonalization, and thus, the reorientation is governed by a competition between the driving force for $\operatorname{TPW}\left(\mathrm{I}_{i j}^{L}(t)\right)$ and the stabilization associated with the delayed viscous adjustment of the rotational bulge (the third term on the right-hand side of equation (13)).

\subsection{Incorporating Stabilization by the Remnant Bulge}

As described in the context of Figure 1c, remnant bulge stabilization arises when the response of the rotating planet to a perturbed centrifugal potential is governed by a planetary model with an elastic lithospheric thickness that is different from the thickness associated with the rotational form of the background (i.e., initial, preloading) state. In the case of Figure 1c, the background state is hydrostatic, but the planetary response to the evolving centrifugal potential is determined by a model with $L T \neq 0$.

The inertia tensor perturbation associated with this scenario can be derived by replacing $k_{f}^{T}(\mathrm{LT})$ in the second term of the right-hand side of equation (9) with the fluid Love number governing the hydrostatic form, $k_{f}^{T, *}$. This yields

$$
\begin{aligned}
\mathrm{I}_{i j}(t)= & \mathrm{I}_{0} \delta_{i j}+\frac{a^{5}}{3 G} k_{f}^{T, *} \Omega^{2}\left(\delta_{i 3}-\frac{1}{3}\right) \delta_{i j} \\
& +\frac{a^{5}}{3 G} k^{T}(t, \mathrm{LT}) *\left\{\left[\omega_{i}(t) \omega_{j}(t)-\frac{1}{3} \omega^{2}(t) \delta_{i j}\right]-\Omega^{2}\left(\delta_{i 3}-\frac{1}{3}\right) \delta_{i j}\right\}+\mathrm{I}_{i j}^{L}(t) .
\end{aligned}
$$

The first two terms on the right-hand side represent the initial, hydrostatic state (Figure 1c1). As in equation (9) (or equation (13)), the third term introduces a delayed, viscous response of the rotational bulge 
to the evolving rotation vector. However, in contrast to this earlier equation, the rotational bulge cannot adjust perfectly to a perturbation in pole position (i.e., in the long-term fluid limit, the two terms involving $\Omega$ on the right-hand side of equation (14) do not cancel) and this noncancelation gives rise to the remnant bulge stabilization. We can rearrange equation (14) by collecting the two terms in $\Omega$. This exercise yields

$$
\begin{aligned}
\mathrm{I}_{i j}(t)= & \mathrm{I}_{0} \delta_{i j}+\frac{a^{5}}{3 G}\left[k_{f}^{T, *}-k_{f}^{T}(\mathrm{LT})\right] \Omega^{2}\left(\delta_{i 3}-\frac{1}{3}\right) \delta_{i j} \\
& +\frac{a^{5}}{3 G} k^{T}(t, \mathrm{LT}) *\left[\omega_{i}(t) \omega_{j}(t)-\frac{1}{3} \omega^{2}(t) \delta_{i j}\right]+\mathrm{I}_{i j}^{\mathrm{L}}(t) .
\end{aligned}
$$

The remnant bulge stabilization is given by the second term of the right-hand side, which is a function of the difference between the fluid $k$ Love number for the two planetary models with and without an elastic lithosphere. Note that if we set $L T=0$, this stabilization disappears.

If we once again apply both the small-s approximation to the s-domain form of the $k^{T}$ Love number (equation (12)) and the convolution theorem to equation (15), we arrive, after some minor rearrangement, at the result

$$
\begin{aligned}
\mathrm{I}_{i j}(t)= & \mathrm{I}_{0} \delta_{i j}+\frac{a^{5}}{3 G} k_{f}^{T}(\mathrm{LT})\left[\omega_{i}(t) \omega_{j}(t)-\frac{1}{3} \omega^{2}(t) \delta_{i j}\right] \\
& +\frac{a^{5}}{3 G}\left[k_{f}^{T, *}-k_{f}^{T}(\mathrm{LT})\right] \Omega^{2}\left(\delta_{i 3}-\frac{1}{3}\right) \delta_{i j} \\
& -\frac{a^{5}}{3 G} k_{f}^{T}(\mathrm{LT}) T_{1}\left[\dot{\omega}_{i}(t) \omega_{j}(t)+\omega_{i}(t) \dot{\omega}_{j}(t)-\frac{2}{3} \omega_{\theta}(t) \dot{\omega}_{\ell}(t) \delta_{i j}\right]+\mathrm{I}_{i j}^{L}(t) .
\end{aligned}
$$

Using equation (16) in the eigenvalue equation (2) yields a time-dependent, nonlinear theory of TPW that incorporates stabilization due to both the delayed, viscous adjustment of the rotational bulge and the remnant bulge. As before, the first two terms on the right-hand side of the equation (the equilibrium form) do not impact the diagonalization, and thus, the reorientation of the rotation axis is governed by a competition between the driving force for TPW $\left(l_{i j}^{L}(t)\right)$ and the stabilizations associated with both the delayed viscous adjustment of the rotational bulge and the remnant bulge (the fourth and third terms on the right-hand side of equation (16), respectively).

In analogy with section 2.3.1, we can use the above equations to derive an expression for the inertia tensor perturbation in the case when the driving load is applied over timescales much longer than those that characterize the viscous adjustment of the rotational bulge (i.e., the equilibrium case). Equation (15) then simplifies to

$$
\begin{aligned}
\mathrm{I}_{i j}(t)= & \mathrm{I}_{0} \delta_{i j}+\frac{a^{5}}{3 G} k_{f}^{T}(\mathrm{LT})\left[\omega_{i}(t) \omega_{j}(t)-\frac{1}{3} \omega^{2}(t) \delta_{i j}\right] \\
& +\frac{a^{5}}{3 G}\left[k_{f}^{T, *}-k_{f}^{T}(\mathrm{LT})\right] \Omega^{2}\left(\delta_{i 3}-\frac{1}{3}\right) \delta_{i j}+\mathrm{I}_{i j}^{L}(t) .
\end{aligned}
$$

We could have also derived this expression by setting the time derivatives of the angular velocity components in equation (16) to zero. The above expression extends equation (10) to incorporate remnant bulge stabilization. In contrast to the earlier result, the final location of a load that is applied and retained for all time is not the equator. Rather, the location is established through a balance between the load forcing and the remnant bulge stabilization. In practical terms, the equilibrium TPW is computed, following equation (2), by diagonalizing the sum of the third and fourth terms on the right-hand side of equation (17).

\subsection{External Loads: The Form of $I_{i j}^{L}(t)$}

The derivations above are general in the sense that they apply to the case of either external or internal loading processes. In the case of the latter, the time-dependent inertia tensor may be input from a simulation of mantle convection (on Earth or other terrestrial planets) and, in this regard, one can make use of the well-established mapping between the components of the inertia tensor and the spherical harmonic components of the gravitational potential at degree 2 [e.g., Matsuyama et al., 2006]. These harmonic components must, of course, include contributions both from mantle buoyancy variations and boundary deformations driven by this buoyancy field.

In the case of external mass loads, one can decompose the load inertia tensor $I_{i j}^{L}(t)$ into terms associated with the mass anomalies and the planetary deformation driven by the mass anomalies. The latter contribution can, in turn, be expressed using viscoelastic load Love number theory, just as we used viscoelastic tidal 
Love numbers to express the deformation induced by a perturbed centrifugal potential. In particular, if we denote the inertia tensor perturbation associated with surface mass redistribution on a rigid Earth as $R_{i j}^{R}(t)$, then the inertia perturbation due to the combined effects of this redistribution and the associated planetary deformation, i.e., $L_{i j}^{L}(t)$, is given by

$$
\mathrm{I}_{i j}^{L}(t)=\left[\delta(t)+k^{L}(t, \mathrm{LT})\right] * \mathrm{I}_{i j}^{R}(t)=\mathrm{I}_{i j}^{R}(t)+k^{L}(t, \mathrm{LT}) * \mathrm{I}_{i j}^{R}(t) .
$$

This relation can be applied in place of $L_{i j}^{L}(t)$ in any of the expressions for the total inertia tensor perturbation derived above when one is considering TPW driven by surface mass loading. In particular, equation (16) becomes

$$
\begin{aligned}
\mathrm{I}_{i j}(t)= & \mathrm{I}_{0} \delta_{i j}+\frac{a^{5}}{3 G} k_{f}^{T}(\mathrm{LT})\left[\omega_{i}(t) \omega_{j}(t)-\frac{1}{3} \omega^{2}(t) \delta_{i j}\right] \\
& +\frac{a^{5}}{3 G}\left[k_{f}^{T, *}-k_{f}^{T}(\mathrm{LT})\right] \Omega^{2}\left(\delta_{i 3}-\frac{1}{3}\right) \delta_{i j}+\left[l_{i j}^{R}(t)+k^{L}(t, \mathrm{LT}) * l_{i j}^{R}(t)\right] \\
& -\frac{a^{5}}{3 G} k_{f}^{T}(\mathrm{LT}) T_{1}\left[\dot{\omega}_{i}(t) \omega_{j}(t)+\omega_{i}(t) \dot{\omega}_{j}(t)-\frac{2}{3} \omega_{t}(t) \dot{\omega}_{\ell}(t) \delta_{i j}\right] .
\end{aligned}
$$

\subsubsection{The Case of an Axisymmetric Surface Mass Load}

It will be useful, in comparing our results to those of Willemann [1984] and Matsuyama et al. [2006], to consider a further special case of equation (19) valid for an axisymmetric surface mass load applied at time $t=0$ and retained for all time. We can denote the space-time dependence of this surface mass load by $R(\theta, \psi) H(t)$, where $\theta$ and $\psi$ are the colatitude and east longitude, respectively, and $H(t)$ is the Heaviside step function. (We note that a disk load with arbitrary time dependence can be modeled as a series of step loadings with suitable time lags, and therefore, in practical terms, the Heaviside time dependence involves no loss of generality.) The spherical harmonic decomposition of $R$ can then be written as

$$
R(\theta, \psi)=\sum_{\ell=0}^{\infty} \sum_{m=-\ell}^{\ell} R_{\ell m} Y_{\ell m}(\theta, \psi),
$$

where $Y_{\ell m}$ are complex surface spherical harmonics of degree $\ell$ and order $m$. We will assume that these basis functions are normalized such that

$$
\int_{S}^{Y_{\ell^{\prime} m^{\prime}}^{\dagger}}(\theta, \psi) Y_{\ell m}(\theta, \psi) \mathrm{d} \Omega=4 \pi \delta_{\ell \ell^{\prime}} \delta_{m m^{\prime}}
$$

where $\uparrow$ denotes complex conjugation.

The load inertia tensor perturbation, $\mathbf{I}^{R}(t)$, can be expressed in terms of the spherical harmonic components of the surface mass load at degree 2 [e.g., Matsuyama et al., 2006]:

$$
\begin{aligned}
& l_{11}^{R}(t)=4 \pi a^{4}\left[\frac{1}{3 \sqrt{5}} R_{20}-\sqrt{\frac{2}{15}} \operatorname{Re}\left(R_{22}\right)\right] H(t) \\
& l_{22}^{R}(t)=4 \pi a^{4}\left[\frac{1}{3 \sqrt{5}} R_{20}+\sqrt{\frac{2}{15}} \operatorname{Re}\left(R_{22}\right)\right] H(t) \\
& l_{33}^{R}(t)=-\frac{8 \pi a^{4}}{3 \sqrt{5}} R_{20} H(t) \\
& l_{12}^{R}(t)=\frac{8 \pi a^{4}}{\sqrt{30}} \operatorname{Im}\left(R_{22}\right) H(t) \\
& l_{13}^{R}(t)=\frac{8 \pi a^{4}}{\sqrt{30}} \operatorname{Re}\left(R_{21}\right) H(t) \\
& l_{23}^{R}(t)=-\frac{8 \pi a^{4}}{\sqrt{30}} \operatorname{Im}\left(R_{21}\right) H(t) .
\end{aligned}
$$

Next, consider an axisymmetric load centered at a colatitude $\theta_{L}$ and east longitude $\psi_{L}$. Let us say that this load, if centered at the north pole, had a degree 2 zonal harmonic given by $R_{20}^{\prime}$ then one can show that the spherical harmonic coefficients for the load centered at $\left(\theta_{L}, \psi_{L}\right)$ are given by [Matsuyama et al., 2006]

$$
R_{2 m}=R_{20}^{\prime} \frac{Y_{2 m}^{\dagger}\left(\theta_{L}, \psi_{L}\right)}{\sqrt{5}}
$$


Willemann [1984] normalized the surface mass load by considering the ratio between the degree 2 perturbations in the geopotential associated with the direct effect of the load and the hydrostatic rotational bulge. In particular, he defined a parameter $Q^{\prime}$ such that

$$
Q^{\prime}=-\frac{\frac{4 \pi a G}{5} R_{20}^{\prime}}{-\frac{1}{3 \sqrt{5}} a^{2} \Omega^{2} k_{f}^{T, *}} .
$$

Using equations (23) and (24) in equation (22), and, for simplicity, choosing $\psi_{L}=0$ yields

$$
\begin{aligned}
& l_{11}^{R}(t)=\frac{\Omega^{2} a^{5}}{9 G} k_{f}^{T, *} Q^{\prime}\left[P_{20}\left(\cos \theta_{L}\right)-\frac{1}{2} P_{22}\left(\cos \theta_{L}\right)\right] H(t) \\
& l_{22}^{R}(t)=\frac{\Omega^{2} a^{5}}{9 G} k_{f}^{T, *} Q^{\prime}\left[P_{20}\left(\cos \theta_{L}\right)+\frac{1}{2} P_{22}\left(\cos \theta_{L}\right)\right] H(t) \\
& l_{33}^{R}(t)=-\frac{2 \Omega^{2} a^{5}}{9 G} k_{f}^{T, *} Q^{\prime} P_{20}\left(\cos \theta_{L}\right) H(t) \\
& I_{13}^{R}(t)=\frac{\Omega^{2} a^{5}}{9 G} k_{f}^{T, *} Q^{\prime} P_{21}\left(\cos \theta_{L}\right) H(t) \\
& l_{12}^{R}(t)=I_{23}^{R}(t)=0,
\end{aligned}
$$

where the $P_{2 m}$ are unnormalized associated Legendre polynomials. Our predictions of time-dependent TPW driven by an axisymmetric surface mass load are generated by applying the above expressions into (19) and diagonalizing the result.

\subsubsection{The Equilibrium Theory of Willemann [1984] and Matsuyama et al. [2006]}

Willemann [1984] and Matsuyama et al. [2006] were concerned with the final displacement of the rotation axis in the case of the axisymmetric loading treated in the last subsection. If we consider the inertia tensor an infinite time after the application of the surface mass load, then equation (19) becomes

$$
\begin{aligned}
\mathrm{I}_{i j}\left(t_{\infty}\right)= & \mathrm{I}_{0} \delta_{i j}+\frac{a^{5}}{3 G} k_{f}^{T}(\mathrm{LT})\left[\omega_{i}\left(t_{\infty}\right) \omega_{j}\left(t_{\infty}\right)-\frac{1}{3} \omega^{2}\left(t_{\infty}\right) \delta_{i j}\right] \\
& +\frac{a^{5}}{3 G}\left[k_{f}^{T, *}-k_{f}^{T}(\mathrm{LT})\right] \Omega^{2}\left(\delta_{i 3}-\frac{1}{3}\right) \delta_{i j}+\left[1+k_{f}^{L}(\mathrm{LT})\right] \mathrm{I}_{i j}^{R}\left(t_{\infty}\right) .
\end{aligned}
$$

The equilibrium TPW is governed by the diagonalization of the third plus fourth term on the right-hand side of this equation. If we denote the sum of these two terms by the superscript eq, then we have

$$
\mathrm{I}_{i j}^{\mathrm{eq}}\left(t_{\infty}\right)=\frac{a^{5}}{3 G}\left[k_{f}^{T, *}-k_{f}^{T}(\mathrm{LT})\right] \Omega^{2}\left(\delta_{i 3}-\frac{1}{3}\right) \delta_{i j}+\left[1+k_{f}^{L}(\mathrm{LT})\right] \mathrm{I}_{i j}^{R}\left(t_{\infty}\right) .
$$

Using equations (23)-(25) in equation (27), and diagonalizing the result, yields a TPW angle $\delta$ given by

$$
\delta=\frac{1}{2} \arctan \left[\frac{Q_{\mathrm{eff}} \sin \left(2 \theta_{L}\right)}{1-Q_{\mathrm{eff}} \cos \left(2 \theta_{L}\right)}\right],
$$

where $Q_{\text {eff }}=Q^{\prime} \alpha$, and

$$
\alpha=\frac{1+k_{f}^{L}(\mathrm{LT})}{1-k_{f}^{T}(\mathrm{LT}) / k_{f}^{T, *}} .
$$

This result for $\delta$ is identical to the expression derived by Matsuyama et al. [2006].

The TPW angle is a function of the initial load colatitude, $\theta_{L}$, and on the parameter $Q_{\text {eff }}$. Embedded in the latter is a sensitivity to the uncompensated size of the load (governed by the parameter $Q^{\prime}$ ) and the parameter $\alpha$, which is a function of the planetary density structure and the elastic thickness of the lithosphere. In Figure 2 we plot the TPW angle versus $\theta_{L}$ for a suite of solutions distinguished on the basis of the choice for $Q_{\text {eff }}$. A detailed discussion of the figure can be found in Matsuyama et al. [2006], and we include it here because it will serve as a useful reference for, and check of, the time-dependent TPW results discussed in the next section. We note that as $Q_{\text {eff }} \rightarrow \infty$ in Figure 2 (or equation (28)), the reorientation of the pole is $90-\theta_{L^{\prime}}$; that is, the final position of the pole is the equator. This is as one would expect. For a very large load, the load driving TPW will dominate the remnant bulge stabilization and the solution converges to the scenario in Figure $1 \mathrm{a} 5$ or $1 \mathrm{~b} 5$ described by Gold [1955]. As an example of a smaller surface mass load, consider the case of $Q_{\text {eff }}=0.5$. A load of this size placed at an initial colatitude of $20^{\circ}$, will ultimately drive TPW of $\sim 13^{\circ}$, or just $\sim 20 \%$ of the $70^{\circ}$ reorientation expected on the basis of an equilibrium theory of Gold [1955]. 


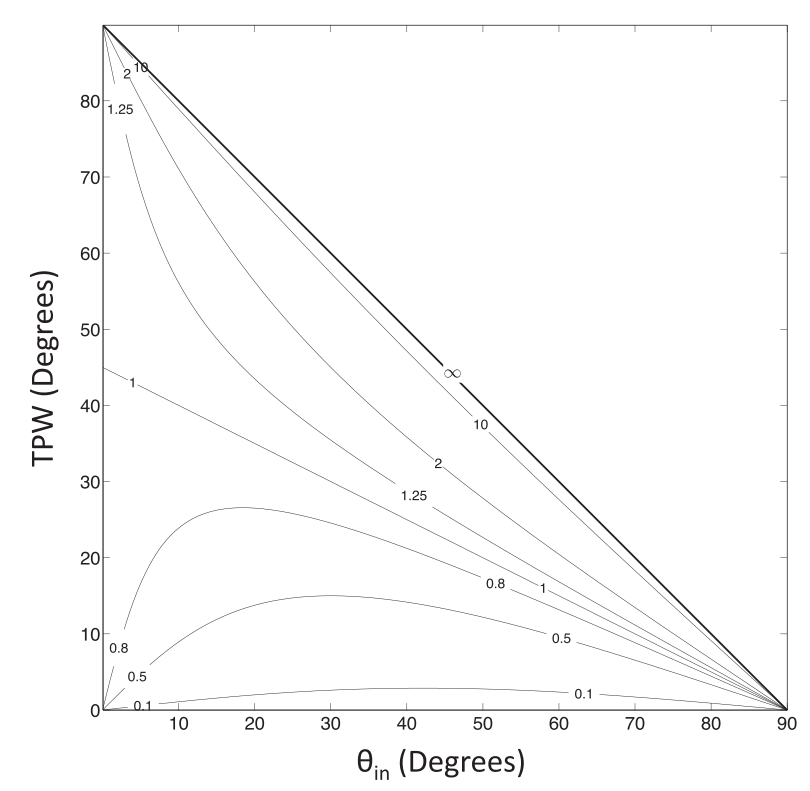

Figure 2. Predictions of equilibrium TPW angle as a function of the initial colatitude $\left(\theta_{L}\right)$ of the loading (see equation (28)). The lines join solutions based on the same value of $Q_{\text {eff }}$, as indicated.

\section{Results}

In this section we present a suite of illustrative predictions of time-dependent TPW on spherical, self-gravitating, Maxwell viscoelastic Earth and Mars models that incorporate stabilization from both the delayed adjustment of the rotational bulge and the remnant bulge. To begin, we consider TPW on Earth driven by simplified surface mass loads.

\subsection{Time-Dependent TPW on Earth: Some Illustrative Examples}

The elastic and density structure of the Earth model is taken from the seismically inferred Preliminary Reference Earth Model [Dziewonski and Anderson, 1981]. The viscosity of the Earth model is discretized into three layers: a uniform elastic (i.e., infinite viscosity) lithosphere of thickness LT; a constant upper mantle viscosity of $5 \times 10^{20} \mathrm{~Pa}$; and a constant lower mantle viscosity denoted by $v_{l m}$. Both LT and $v_{l m}$ serve as free parameters of the modeling, but our standard case will be characterized by LT $=15 \mathrm{~km}$ and $v_{l m}=10^{22} \mathrm{~Pa}$ s. The external load will be modeled as an axisymmetric disk load placed on the Greenwich meridian, and at an initial colatitude of $\theta_{L}$. The standard case will adopt $\theta_{L}=45^{\circ}$ and a $Q_{\text {eff }}$ value of unity. The size of the load is assumed to increase from zero to the adopted $Q_{\text {eff }}$ value in the first 1 Myr of each simulation with a time dependence given by a hyperbolic tangent function. We chose this timescale because it is consistent with the underlying assumption of the theory that the loading timescale is longer than the longest normal mode decay time, yet it is sufficiently short that the computed TPW departs from the path predicted using an equilibrium theory. Our predictions are based on equation (19), with the inertia tensor perturbation associated with the direct effect of the surface mass load given by equation (25). The Earth has a broken lithosphere, and, as discussed in section 1, we interpret the parameter LT as an effective elastic lithospheric thickness; our choice of $L T=15 \mathrm{~km}$ for the standard case is likely above the upper bound for this thickness [Creveling et al., 2012], and thus, in an analysis described below we will consider the sensitivity of the predictions to a variation of LT in the range 5-25 km. As a final point, a numerical instability occurs for time steps that are too short (see Appendix A). In this regard, our time step for each model run is chosen to be uniform and as small as possible without encountering this instability.

In Figure 3, we plot the prediction of time-dependent TPW based on the standard case. The large dot on the extreme right of the figure is the equilibrium TPW computed using the analytic expression (28) [Matsuyama et al., 2006]. The agreement between this analytic expression and the long-time asymptote of the time-dependent TPW prediction provides an important check on the latter. The time-dependent prediction reaches $50 \%$ and $90 \%$ of the equilibrium value $\left(22.5^{\circ}\right)$ in $6.1 \mathrm{Myr}$ and $18.3 \mathrm{Myr}$, respectively. (We henceforth denote the time elapsed for the pole to reach half its maximum value by the symbol $t_{1 / 2}$ ). 


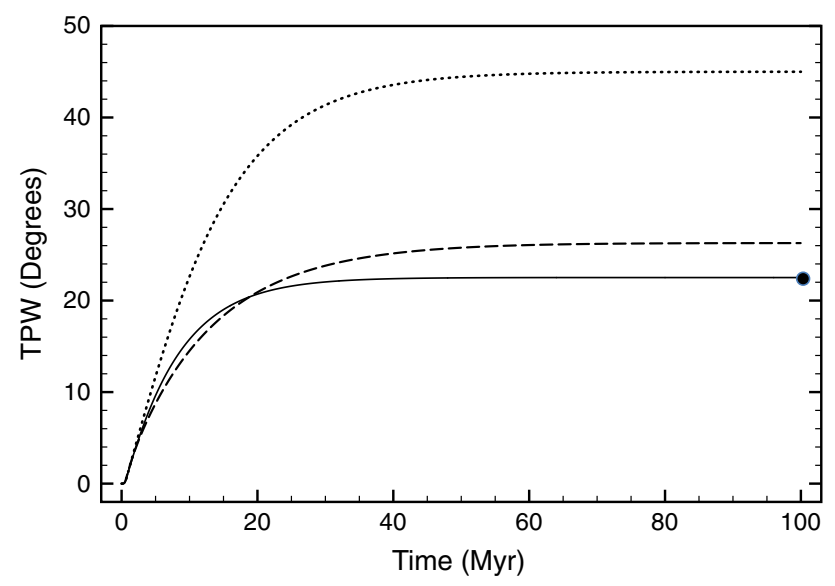

Figure 3. Predictions of surface load induced TPW as a function of time computed using a Maxwell viscoelastic Earth characterized by an elastic lithospheric thickness $\mathrm{LT}=15 \mathrm{~km}$, and upper and lower mantle viscosities of $5 \times 10^{20} \mathrm{~Pa} \mathrm{~s}$ and $10^{22} \mathrm{~Pa} \mathrm{~s}$, respectively. The surface load is an axisymmetric disk centered at an initial colatitude $\theta_{L}=45^{\circ}$. The disk size is increased such that the parameter $Q_{\text {eff }}$ is varied from 0.0 to 1.0 in the first 1 Myr of the simulation (see text) and then retained for all time. This is the test model case discussed in the text. The solid and dotted lines are solutions in which remnant bulge stabilization is either included (equation (19) with $\mathbf{I}^{R}(t)$ given by equation (25)) or not included (the same equations, with the exception that the third term on the right-hand side of equation (19) is deleted), respectively, in the TPW calculation. As a check on the result with the remnant bulge stabilization (solid line), we have repeated that calculation using the linearized rotational stability theory derived by Chan et al. [2011] and discussed in section 2.1 (dashed line; see text). All calculations incorporate stabilization by the delayed, viscous adjustment of the rotational bulge. The solid circle on the right-hand side of the figure is the TPW predicted for the test model case using the equilibrium (i.e., infinite time) theory of Matsuyama et al. [2006] (see equation (28)).

As a second check on the result in Figure 3, we have repeated the standard calculation using the linearized rotational stability theory derived by Chan et al. [2011] and discussed in section 2.1 (dashed line, Figure 3). This theory does not require that the forcing have a timescale comparable to or longer than the longest mode of viscoelastic decay, but it assumes that perturbations to the pole position are "small." Chan et al. [2011] explored the range of validity for their linearized theory and found that, for forcing placed near midlatitudes, errors are only $\mathrm{O}(10 \%)$ for TPW of $20-30^{\circ}$. This estimate is consistent with the offset between the solid and dashed lines in Figure 3 at the limit of $t \rightarrow \infty$. We assume that in the earliest stages of TPW in Figure 3, the Chan et al. [2011] theory is more accurate. During this phase, we note that the discrepancy between results generated with the linear and nonlinear theories is small.

The dotted line in Figure 3 is analogous to the solid, with the exception that stabilization due to the remnant bulge is ignored by deleting the third term on the right-hand side of equation (19). This deletion yields a governing equation consistent with the rotational stability theory derived by Ricard et al. [1993]. The equilibrium TPW predicted in this case $\left(45^{\circ}\right)$ is such that the final position of the disk load is at the equator, as expected from the physics associated with Figure 1b, Gold [1955], and Ricard et al. [1993]. As we noted in our discussion of stabilization due to the remnant bulge, the existence of even a relatively thin elastic lithosphere is sufficient to significantly reduce the predicted TPW from the value expected on the basis of the canonical theory of Gold [1955] [Matsuyama et al., 2006; Daradich et al., 2008]. However, we note from Figure 3 that the inclusion of remnant bulge stabilization also acts to alter the characteristic timescale of the predicted TPW. As an example, $t_{1 / 2}=9.9 \mathrm{Myr}$ for the dotted line in Figure 3 , which is $65 \%$ longer than the analogous timescale for the case where the remnant bulge is included. This difference is primarily due to the reduced amplitude of TPW when remnant bulge stabilization is included, whereas the damping caused by the viscous rotational bulge remains relatively unchanged between both cases.

We next turn to a sensitivity analysis in which we repeat the calculation in Figure 3 (solid line) for a suite of simulations in which $Q_{\text {eff }}, v_{l m}$, and $\theta_{L}$ are individually varied while the other parameters are held constant at values that define the standard case. Figures 4 and 5 show predictions of time-dependent TPW and TPW 

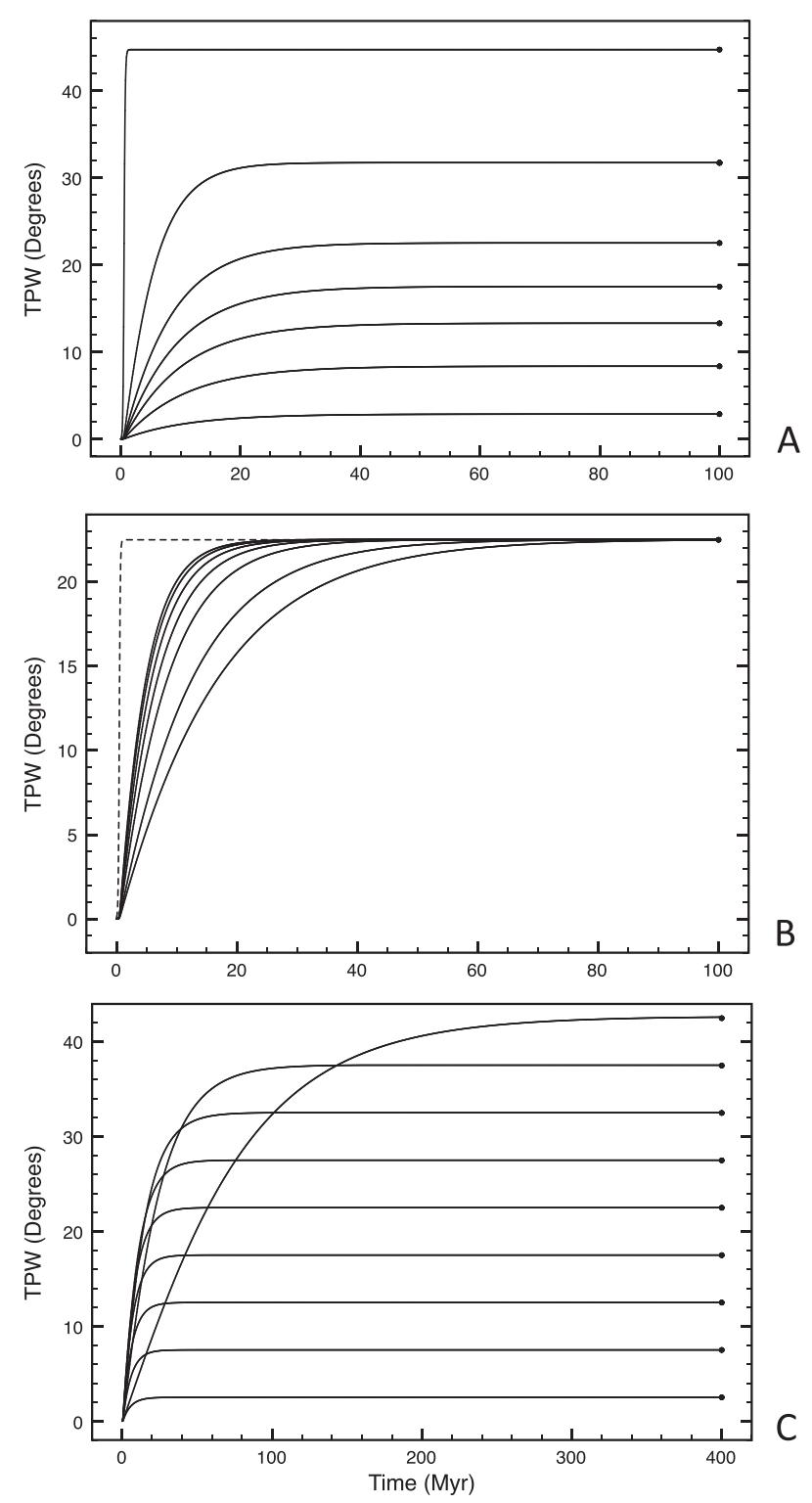

Figure 4. Predictions of surface load induced TPW as a function of time computed for a suite of simulations in which the parameter $Q_{\text {eff }}$, the lower mantle viscosity $v_{l m^{\prime}}$ or the initial load colatitude $\theta_{L}$ are varied from the test model case used in Figure 3 (solid line). (a) $Q_{\text {eff }}$ values (from bottom to top) of $0.1,0.3,0.5,0.7,1.0,2.0$, and 100.0. (b) $v_{l m}$ values (solid lines from left to right) of $1,2,3,5,10,20$, and $30 \times 10^{21} \mathrm{~Pa}$ s. The dashed line is a solution in which the rotational bulge stabilization is excluded (i.e., the term involving $T_{1}$ in equation (19) is set to 0). (c) $\theta_{L}$ values (from top right to bottom right) of $5^{\circ}, 15^{\circ}, 25^{\circ}, 35^{\circ}, 45^{\circ}, 55^{\circ}, 65^{\circ}, 75^{\circ}$, and $85^{\circ}$. All calculations include stabilization by both the delayed viscous adjustment of the rotational bulge and the remnant bulge (equation (19) with $\mathbf{I}^{R}(t)$ given by equation (25)). The solid circles on the right-hand side of the figure are the TPW predictions based on the equilibrium (i.e., infinite time) theory of Matsuyama et al. [2006] (see equation (28)).

speed, respectively. In addition, for each of these time-dependent simulations, we compute and plot the value of $t_{1 / 2}$ and the peak TPW speed in Figures 6 and 7.

As $Q_{\text {eff }}$ is increased, the equilibrium position of the pole increases asymptotically toward $45^{\circ}$. That is, as the load size increasingly dominates the remnant bulge stabilization, the final load position will reach progressively closer to the equator (Figure 4a). (Note, once again, that the long time limit of each simulation in Figure 4a matches the equilibrium TPW predicted on the basis of equation (28).) Regardless of the value of $Q_{\text {eff }}$, the peak speed of TPW is obtained at the end of the loading phase, 1 Myr after the onset of loading. It is interesting to note that the peak TPW speed is a linear function of the adopted value of $Q_{\text {eff }}$ (Figure 7a). In contrast, the time to reach half the final TPW, $t_{1 / 2}$, varies in a more complex manner with the adopted $Q_{\text {eff }}$ 

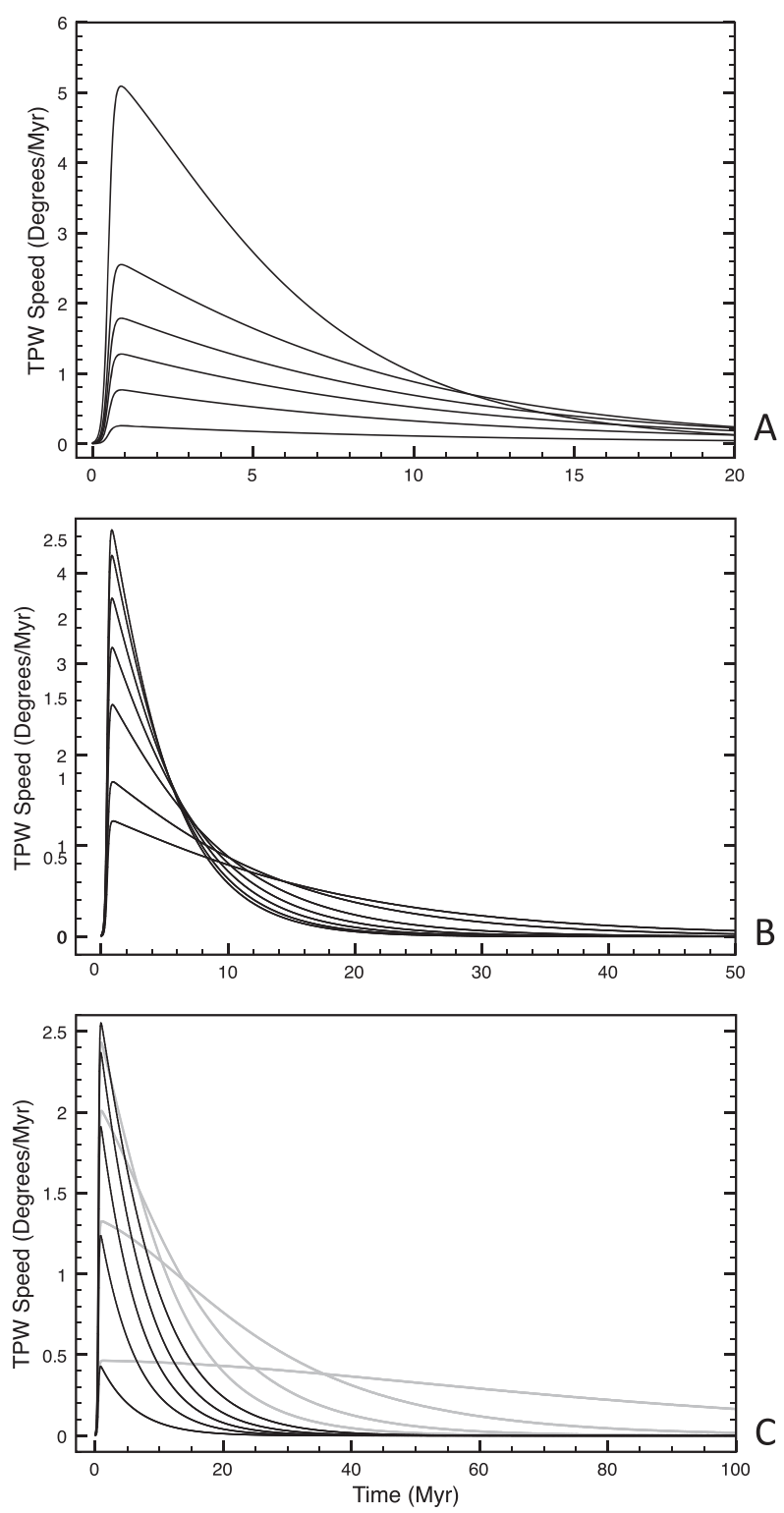

Figure 5. Predictions of surface load induced TPW speed as a function of time computed for a suite of simulations in which the parameter $Q_{\text {eff }}$, the lower mantle viscosity $v_{l m}$, or the initial load colatitude $\theta_{L}$ are varied from the test model case used in Figure 3 (solid line). (a) $Q_{\text {eff }}$ values (from bottom to top) of $0.1,0.3,0.5,0.7,1.0$, and 2.0. (b) $v_{l m}$ values (from top to bottom at location of peaks) of 1, 2, 3, 5, 10,20 , and $30 \times 10^{21} \mathrm{~Pa}$ s. (c) $\theta_{L}$ values (from bottom to top at peak values of grey lines) of $5^{\circ}, 15^{\circ}, 25^{\circ}, 35^{\circ}$, and (from top to bottom at peak values of black lines) $45^{\circ}, 55^{\circ}, 65^{\circ}, 75^{\circ}$, and $85^{\circ}$. All calculations include stabilization by both the delayed viscous adjustment of the rotational bulge and the remnant bulge (equation (19) with $\mathbf{I}^{R}(t)$ given by equation (25)).

(Figure 6a). Specifically, for values of $Q_{\text {eff }} \leq 0.5, t_{1 / 2} \sim 7-8 \mathrm{Myr}$ and is relatively insensitive to variations in $Q_{\text {eff }}$. As $Q_{\text {eff }}$ increases above $\sim 0.5, t_{1 / 2}$ decreases more rapidly.

Each of Figures $4 b, 5 b, 6 b$, and $7 b$ shows results for a suite of simulations in which the lower mantle viscosity of the Earth model is varied from $10^{21} \mathrm{~Pa} s$ to $3 \times 10^{22} \mathrm{~Pa}$ s. The equilibrium (i.e., long-time) position of the pole is entirely insensitive to $v_{l m}$, while the time-dependent trajectory toward this equilibrium value is not (Figure $4 \mathrm{~b}$ ). The parameter $t_{1 / 2}$ is linearly proportional to the adopted value of $v_{l m}$ (Figure $6 \mathrm{~b}$ ). The proportionality is such that the factor of 30 increase in viscosity treated in the figure produces a factor of $\sim 3.5$ increase in the time necessary for the pole to be displaced by half its equilibrium displacement. Figure $6 \mathrm{~b}$ shows the analogous prediction for $Q_{\text {eff }}=0.1$. This additional calculation indicates that the linear proportionality between $v_{l m}$ and $t_{1 / 2}$ is a general result and that the proportionality constant connecting these 

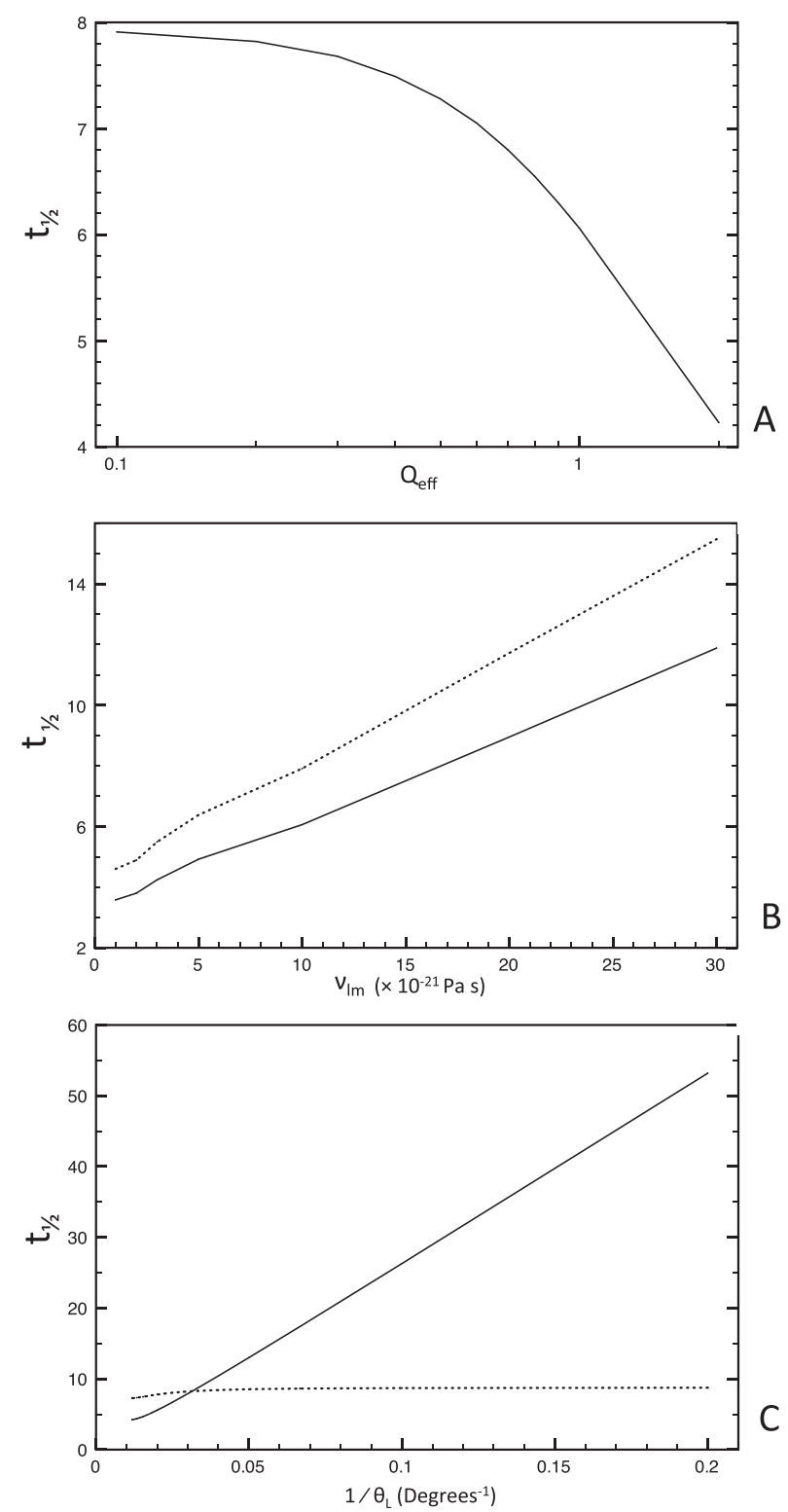

Figure 6. Solid lines on each frame show predictions of the time (in Myr) required for the pole to be displaced by half the maximum (i.e., infinite time) value $\left(t_{1 / 2}\right)$ for a suite of simulations in which the parameter $Q_{\text {eff, }}$ the lower mantle viscosity $v_{l m}$, or the initial load colatitude $\theta_{L}$ are varied from the test model case used in Figure 3 (solid line). (a) $Q_{\text {eff }}$ is varied from 0.1 to 2.0 ; (b) $v_{l m}$ is varied from $10^{21} P a s$ to $30 \times 10^{21} \mathrm{~Pa} \mathrm{~s}$; and (c) $\theta_{L}$ is varied from $5^{\circ}$ (or $1 / \theta_{L}=0.2$ ) to $85^{\circ}$. In Figures $6 \mathrm{~b}$ and $6 \mathrm{c}$, the dotted line shows a result analogous to the solid line case (where $Q_{\text {eff }}=1.0$ ) with the exception that the parameter $Q_{\text {eff }}$ has a value of 0.1 . All calculations include stabilization by both the delayed viscous adjustment of the rotational bulge and the remnant bulge (equation (19) with $\mathbf{I}^{R}(t)$ given by equation (25)).

parameters is weakly sensitive to the adopted $Q_{\text {eff }}$. In contrast to these results, while the peak TPW speed decreases monotonically with increasing $v_{l m}$, this decrease is not a linear function of the adopted lower mantle viscosity (Figure 7b).

The dashed line in Figure 4b represents a TPW simulation in which the stabilizing effects of the delayed adjustment of the rotational bulge is excluded. At each stage in the loading, the dashed line represents the TPW computed using the equilibrium theory of Matsuyama et al. [2006] (equation (28)). The lag between the dashed line and each of the solid lines represents the delay associated with the adjustment of the rotational bulge, which is, of course, a function of the adopted lower mantle viscosity. Inferences of lower mantle viscosity [Nakada and Lambeck, 1989; Mitrovica, 1996; Lambeck et al., 1998; Mitrovica and Forte, 2004] are greater than the lowest viscosity $\left(10^{21} \mathrm{~Pa} \mathrm{~s}\right)$ in the figure, indicating that stabilization associated with the rotational bulge is active for loading timescales of order 1 Myr. 

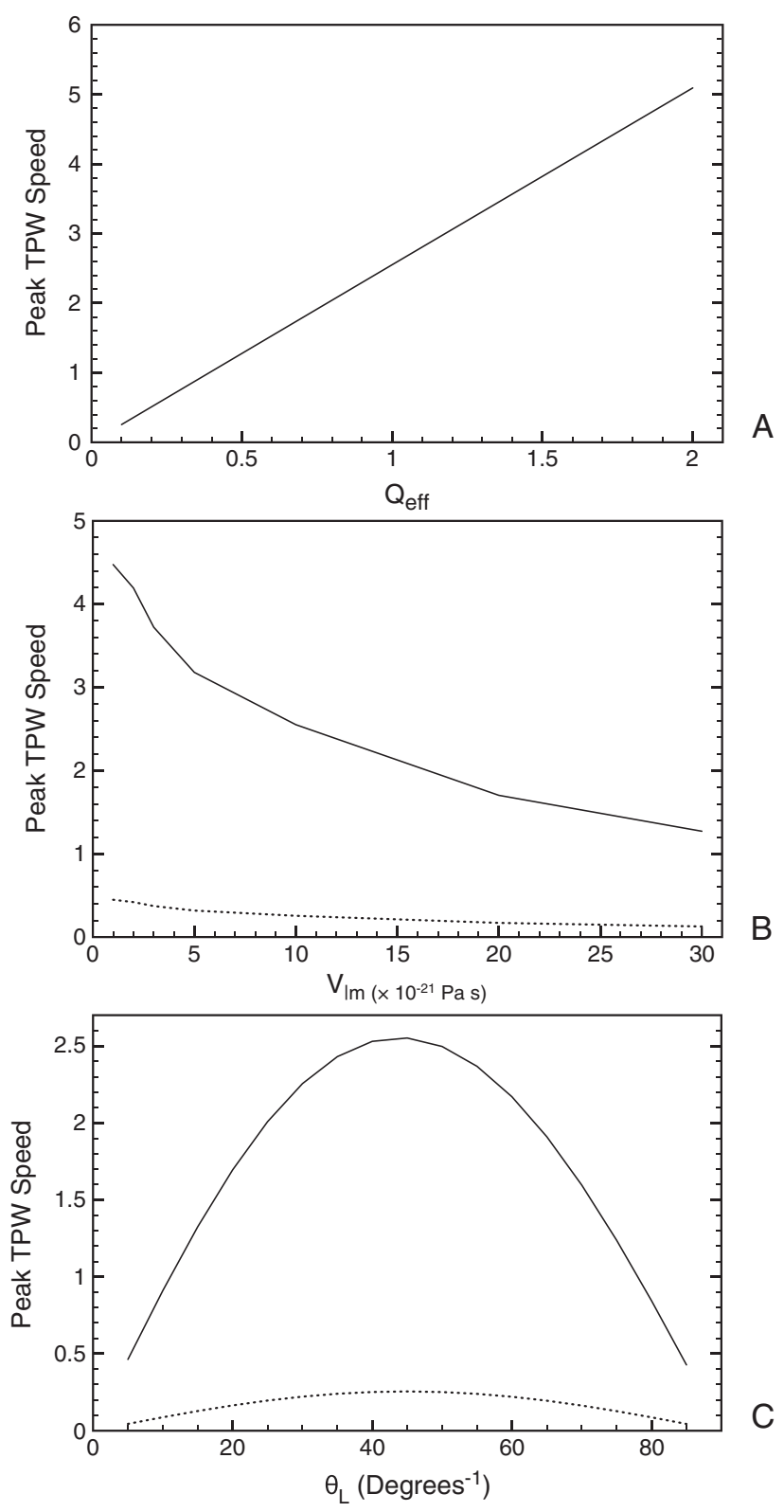

Figure 7. As in Figure 6, except for predictions of the peak TPW speed (Degrees/Myr).

The predicted time dependence of TPW is a complex function of the initial position of the disk load (Figure 4c). This complexity is particularly evident in the time dependence of the TPW speed, which is characterized by a nonmonotonic peak amplitude (Figure 5c). In particular, the predicted peak TPW speed is highest for a load placed at midlatitudes and drops off symmetrically as the initial position of the disk load is placed closer to either the rotation axis ( $\theta_{L}$ is small) or the equator $\left(\theta_{L} \rightarrow 90^{\circ}\right)$ (Figures $5 \mathrm{c}$ and $7 \mathrm{c}$ ). In the latter case, the final TPW is small because the load is already close to the equator (Figure 4c), and thus, the TPW speed is limited by this proximity (e.g., see the $\theta_{L}=85^{\circ}$ case in Figure $5 \mathrm{c}$ ). The explanation for the former, small $\theta_{L}$ case, is more complicated. In the former case, when the load is placed close to the rotation axis, there is a near cancellation between the load-induced forcing and the remnant bulge stabilization since $Q_{\text {eff }}=1$, i.e., the net forcing is small. In this case, even though the total displacement of the pole is large (the equilibrium TPW is $\sim 45^{\circ}$; Figures 2 and $4 \mathrm{c}$ ), the TPW speed will be small throughout the reorientation of the pole from its initial to final position. 


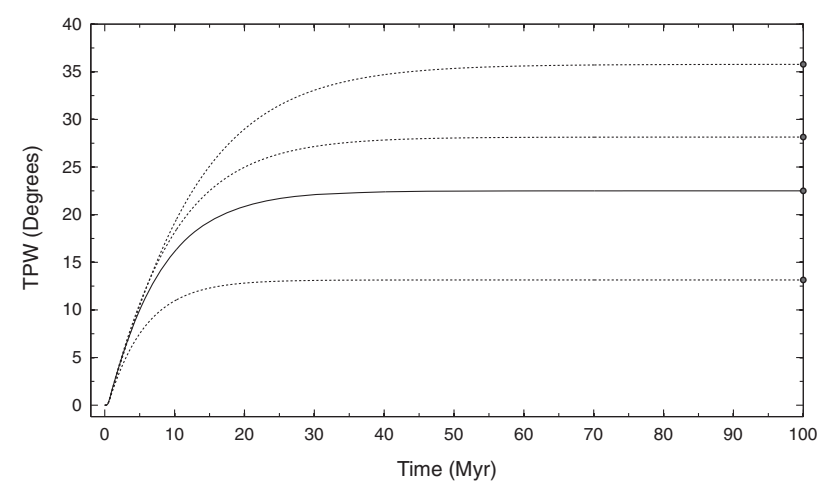

Figure 8. Predictions of surface load induced TPW as a function of time computed using a Maxwell viscoelastic Earth characterized by upper and lower mantle viscosities of $5 \times 10^{20} \mathrm{~Pa}$ s and $10^{22} \mathrm{~Pa} \mathrm{~s}$, respectively. The surface load is an axisymmetric disk centered at an initial colatitude $\theta_{L}=45^{\circ}$. All calculations use the full rotational stability theory in which stabilization by both the delayed, viscous adjustment of the rotational bulge and the remnant bulge are included (equation (19) with $\mathbf{I}^{R}(t)$ given by equation (25)). Solid line: An elastic lithospheric thickness of $\mathrm{LT}=15 \mathrm{~km}$, and a disk size that is increased such that the parameter $Q_{\text {eff }}$ is varied from 0.0 to 1.0 in the first $1 \mathrm{Myr}$ of the simulation (see text) and then retained for all time. This is the test model case discussed in the text. Dashed lines (from top to bottom): LT $=5 \mathrm{~km}, 10 \mathrm{~km}$, and $25 \mathrm{~km}$; for these cases, the uncompensated sizes of the loads ( $Q^{\prime}$; equation (24)) are varied such that the product $Q^{\prime}\left(1+k_{f}^{L}\right)$ remains identical to that used to generate the solid line. The solid circles on the right-hand side of the figure are the equilibrium TPW predicted for these cases (see equation (28)).

In the $Q_{\text {eff }}=0.1$ case (Figure 7c), the net TPW is small because the remnant bulge is stronger than the load-induced forcing (Figure 2), and thus, the TPW speed is limited by the small net displacement of the pole. The symmetry evident in Figure $7 \mathrm{c}$ is particularly interesting given that the equilibrium TPW for these $Q_{\text {eff }}$ values in Figure 2 do not both exhibit symmetry across midlatitudes. The above arguments suggest that the symmetry evident in Figure 7c will disappear for $Q_{\text {eff }}$ values progressively greater than 1 .

As a final point in regard of Figure 4c, the fact that the TPW predicted in the long-time limit is linearly related to $\theta_{L}$ is consistent with the $Q_{\text {eff }}=1$ case in Figure 2 . Note, once again, that the time-dependent TPW predictions in Figure 4c converge to the equilibrium values given by the analytic expression (28).

In Figure 8 we extend the analyses described above to consider the sensitivity of the predictions to a change in the elastic thickness of the lithosphere, LT. The solid line in the figure is the TPW predicted for the standard case, and it is reproduced from Figure 3. As discussed above, this calculation adopts a value of $Q_{\text {eff }}=1.0$. To isolate the impact of $L T$ on the adjustment of the rotational bulge from its direct impact on the load via isostatic compensation, we vary LT from 5 to $25 \mathrm{~km}$ (dashed lines in Figure 8) while holding the product $Q^{\prime}\left(1+k_{f}^{L}\right)$ constant and equal to the $L T=15 \mathrm{~km}$ case. Following our definition for $Q_{\text {eff }} \equiv Q^{\prime} \alpha$ (where $\alpha$ is defined in equation (29)), keeping $Q^{\prime}\left(1+k_{f}^{L}\right)$ constant requires that we vary $Q_{\text {eff }}$ appropriately. As a result, the long-time limits of the TPW predictions are distinct, and they converge to the equilibrium values expected on the basis of equation (28). A more significant difference is the timescale over which

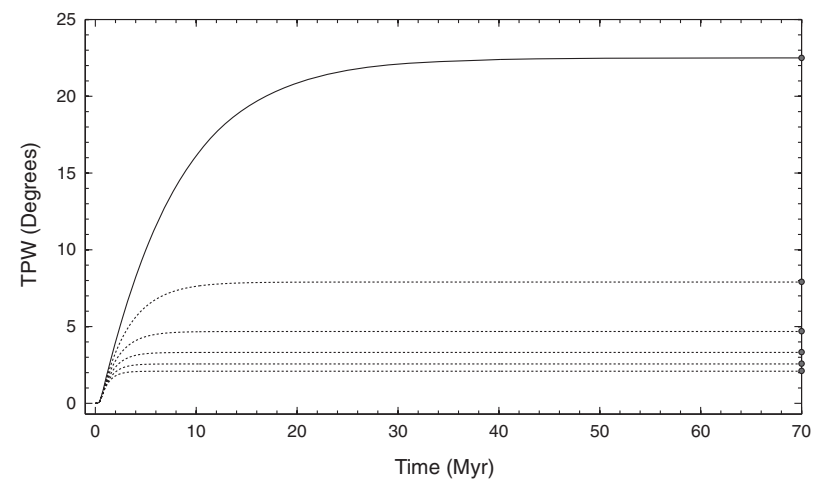

Figure 9. Predictions of TPW as a function of time using the same test model case corresponding to the solid line in Figure 3 (reproduced here also as solid line), except the remaining lines, which have excess ellipticity added to their degree- $2 k$ tidal Love numbers. From top to bottom (dashed lines), the added excess ellipticity values are $0.002,0.004,0.006,0.008$, and 0.01 , respectively. The solid circles on the right-hand side of the figure are the equilibrium TPW predicted for these cases (see equation (28)). 


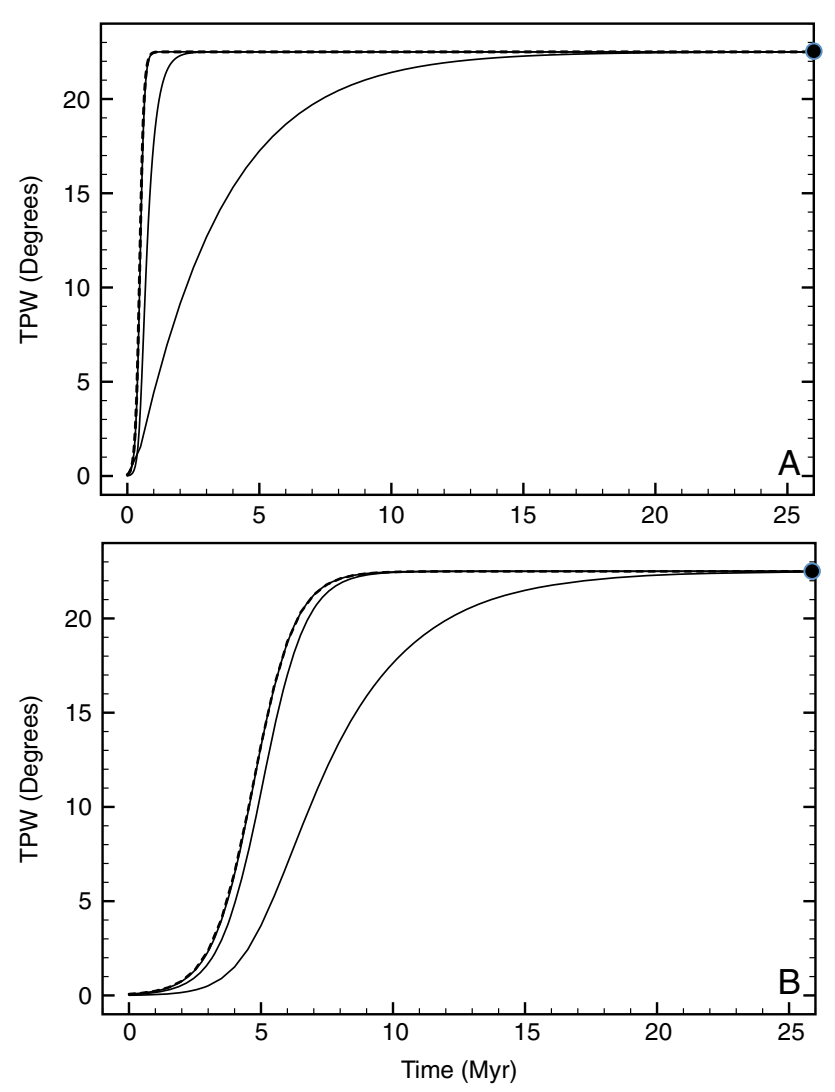

Figure 10. Predictions of surface load induced TPW as a function of time computed using a Maxwell viscoelastic Mars model characterized by an elastic lithospheric thickness LT $=110 \mathrm{~km}$, and mantle viscosities of $10^{21} \mathrm{~Pa} \mathrm{~s}, 10^{22} \mathrm{~Pa}$ s, and $10^{23} \mathrm{~Pa} \mathrm{~s}$ (solid curves from left to right, respectively). The surface load is an axisymmetric disk centered at an initial colatitude $\theta_{L}=45^{\circ}$. The disk size is increased such that the parameter $Q_{\text {eff }}$ is varied from 0.0 to 1.0 in (a) the first 1 Myr and (b) the first 10 Myr of the simulation and then retained for all time. The dashed line on each frame is a solution in which the rotational bulge stabilization is excluded (i.e., the term involving $T_{1}$ in equation (19) is set to 0). The solid circle on the right-hand side on each frame is the TPW predicted using the equilibrium (i.e., infinite time) theory of Matsuyama et al. [2006] (see equation (28)).

these predictions converge to their respective equilibrium values. In particular, as LT increases from $5 \mathrm{~km}$ to $25 \mathrm{~km}, t_{1 / 2}$ decreases from $\sim 9$ Myr to $\sim 4$ Myr. As mentioned in the discussion of Figure 3, this trend is primarily due to the reduced amplitude of TPW associated with the remnant bulge stabilization (third term on the right-hand side of equation (19)). The effect of this stabilization on the perturbed pole position is constant, whereas the stabilization associated with the delayed adjustment of the viscous rotational bulge (fifth term of equation (19)) evolves with time. Given a comparable effective loading $\left(Q^{\prime}\left(1+k_{f}^{L}\right)\right)$, the timescale of the TPW predictions in Figure 8 depends on the time-evolving relative importance of these effects.

In addition to the remnant bulge, a planet may also have a stable excess ellipticity arising, for example, from long timescale convective flow in the interior. On present-day Earth, this excess ellipticity is observed to contribute an additional $\sim 0.01$ to the degree- $2 k$ tidal Love number when averaged over the two principal equatorial axes [Chambat et al., 2010]. Figure 9 shows the stabilizing effect of excess ellipticity on our standard scenario. An excess ellipticity of 0.002 (topmost dashed line), or $20 \%$ of Earth's present-day value, reduces the final TPW by over $60 \%$ relative to the case without this stabilization. The reduction is over $90 \%$ if we adopt the Earths present-day excess ellipticity (bottommost dashed line). It is important to note that this level of stabilization arises from the combined effects of the remnant bulge and the excess ellipticity. Even in the absence of an elastic lithosphere, any long-term excess ellipticity would still significantly stabilize a planets rotation [Creveling et al., 2012].

\subsection{Time-Dependent TPW on Mars: Some Illustrative Examples}

Figure 10 shows a set of illustrative simulations of time-dependent TPW on Mars. The predictions are once again based on equation (19), with the inertia tensor perturbation associated with the direct effect of the surface mass load given by equation (25). The elastic and density structure of the Mars model is taken from 
Zharkov and Gudkova [2005]. The viscosity of the model is discretized into two layers: a uniform elastic lithosphere of thickness $110 \mathrm{~km}$ and constant mantle viscosities of $10^{21} \mathrm{~Pa} \mathrm{~s}, 10^{22} \mathrm{~Pa} \mathrm{~s}$, and $10^{23} \mathrm{~Pa} \mathrm{~s}$ (the three solid lines on each frame). In all cases, the axisymmetric load is placed at $\theta_{L}=45^{\circ}$. In Figure 10 a, the size of this load is assumed to increase from zero to $Q_{\text {eff }}=1$ in the first 1 Myr of each simulation, as in Figures 3-8. For a convective forcing, this is unrealistically short, and therefore, in Figure 10b, the timescale is increased by an order of magnitude.

In each frame of the figure, the dashed line represents a simulation in which the stabilizing effect of the delayed adjustment of the rotational bulge is excluded. As discussed in relation to Figure $4 \mathrm{~b}$, at each stage in the loading, the dashed line represents the TPW computed using the equilibrium theory of Matsuyama et al. [2006] (equation (28)). For loading timescales of either 1 or 10 Myr, stabilization associated with the rotational bulge in the case of a mantle viscosity of $10^{21} \mathrm{~Pa} s$ introduces a negligible lag in the TPW prediction relative to this equilibrium theory. Conversely, this lag only becomes significant over this timescale of loading when the mantle viscosity exceeds $10^{22} \mathrm{~Pa}$ s. The viscosity of Martian mantle is not well constrained, but most recent studies adopt values from $\sim 10^{19} \mathrm{~Pa} \mathrm{~s}$ to $10^{21} \mathrm{~Pa} \mathrm{~s}$, where the range reflects uncertainties associated with both temperature and composition [e.g., Hauck and Phillips, 2002; Williams and Nimmo, 2004; Breuer and Spohn, 2006]. This suggests, with reference to Figure 10, that stabilization associated with the Martian rotational bulge is unimportant for loads that evolve over timescales longer than $\sim 1 \mathrm{Myr}$.

\section{Final Remarks}

We have derived and applied a nonlinear theory for predicting time-dependent TPW of terrestrial planets subject to (internal or external) mass redistribution. The theory accounts for stabilization associated with both the delayed viscous readjustment of the rotational bulge and TPW-induced stresses in an elastic lithosphere (i.e., a remnant bulge). Our derivation is an extension of the treatment by Ricard et al. [1993] to include remnant bulge stabilization and it assumes, following that work, that inertia tensor perturbations have a timescale longer than the viscous response of the planetary model to an applied tidal-effective forcing. Our derivation may also be understood as an extension of the equilibrium rotational stability theory of Willemann [1984] and Matsuyama et al. [2006] to incorporate time dependence.

Previous work has shown that the remnant bulge can significantly reduce the amplitude of TPW relative to calculations which incorporate stabilization by the viscoelastically deforming rotational bulge alone [Willemann, 1984; Matsuyama et al., 2006; Daradich et al., 2008]. Our new calculations demonstrate that the remnant bulge also acts to reduce the characteristic timescale of the TPW (e.g., Figures 3 and 8). This reduction in timescale can be understood by inspection of the equations governing TPW; namely, the eigenvalue problem in equation (2) with the total inertia tensor perturbation given by equation (16). The remnant bulge stabilization is embodied within the third term on the right-hand side of the latter equation. This term depends on the thickness of the elastic lithosphere and the original orientation of the rotation axis, and it is stationary in time. Thus, if stabilization by the remnant bulge is switched on (as in Figure 3), or if it is strengthened by increasing the thickness of the elastic lithosphere (as in Figure 8), then it will moderate the time dependence associated with the delayed viscous adjustment of the rotational bulge (the fourth term on the right-hand side of equation (16)).

The remaining calculations based on the Heaviside-type loading of a viscoelastic Earth model reveal other interesting sensitivities. For example, the peak TPW speed is linearly dependent on the effective load size, $Q_{\text {eff }}$, while the time to reach half the final (equilibrium) displacement of the pole, $t_{1 / 2}$, is linearly proportional to the adopted lower mantle viscosity. Also, the peak TPW speed decreases monotonically with increasing $v_{l m}$, but this decrease is not a linear function of $v_{l m}$.

Finally, using current estimates of mantle viscosity for both Earth and Mars, our calculations suggest that the transient stabilization associated with the rotational bulge will be significant for Earth but negligible for Mars in the case of forcings with timescale 1 Myr or longer. That is, the predicted TPW on Mars driven by forcings over these timescales will track the equilibrium response, where the latter includes stabilization associated with the remnant bulge [Willemann, 1984; Matsuyama et al., 2006]

The calculations presented in the last section all converge, as they should, to the equilibrium solution a long time after the application of the loading. In future work we will further extend the theory described here to incorporate a viscoelastic, rather than elastic, lithosphere. In this case, TPW should trend toward the 
equilibrium theory discussed by Willemann [1984] and Matsuyama et al. [2006] over timescales that are long, but shorter than the viscous relaxation time of the viscoelastic lithosphere. However, once the timescale of the loading exceeds this relaxation time, stabilization by the remnant bulge will disappear, and the pole will further reorient so as to bring the load to the equator, as predicted by the classic equilibrium theory of Gold [1955].

\section{Appendix A: A Note on Time Step Sizes in the Numerical Implementations}

As mentioned in section 2.1, the eigenvalue approach discussed in this article is based on the assumption that the timescale of loading is similar to or longer than the reorientation timescale of the rotation pole. A further long timescale condition was imposed in equation (12) (following Ricard et al. [1993]).

In numerically implementing the theory described in sections 2.3 and 2.4 , we have (empirically) found quantifiable limitations consistent with such long timescale assumptions. Consider the principal axis associated with the maximum moment of inertia of the viscous part of the rotational bulge (whose adjustment is induced by the rotational-potential perturbation but delayed by viscosity, i.e., the second and fourth term on the right-hand side of equation (16)), and the maximum moment principal axis of the load (last term of equation (16)). The numerical solution will only converge if both of these axes are on the same side of the rotation pole in the previous time step (for the initial step, this is $\boldsymbol{\Omega}$ ), which only happens when sufficiently large time steps are taken. The exact sizes of such time steps depend on the planetary model used (which affect $k_{f}^{T}$ and $T_{1}$ ). This limitation could be thought of as a requirement to leave enough time between time steps for the viscous portion of the rotational bulge to begin adjusting toward the latest perturbation of the rotational potential, thus inherently satisfying the long timescale assumptions mentioned above.

\section{Acknowledgments}

We thank two anonymous reviewers for their comprehensive and constructive reviews. We acknowledge funding from Harvard University and the Canadian Institute for Advanced Research. This work is also supported in part by the M. Hildred Blewett Fellowship of the American Physical Society, www.aps.org.

\section{References}

Breuer, D., and T. Spohn (2006), Viscosity of the Martian mantle and its initial temperature: Constraints from crust formation history and the evolution of the magnetic field, Planet. Space Sci., 54(2), 153-169, doi:10.1016/j.pss.2005.08.008.

Cambiotti, G., Y. Ricard, and R. Sabadini (2011), New insights into mantle convection true polar wander and rotational bulge readjustment, Earth Planet. Sci. Lett., 310(3-4), 538-543, doi:10.1016/j.epsl.2011.08.009.

Chambat, F., Y. Ricard, and B. Valette (2010), Flattening of the Earth: Further from hydrostaticity than previously estimated, Geophys. J. Int., 183(2), 727-732, doi:10.1111/j.1365-246X.2010.04771.x.

Chan, N.-H., J. X. Mitrovica, I. Matsuyama, J. R. Creveling, and S. Stanley (2011), The rotational stability of a convecting Earth: Assessing inferences of rapid TPW in the late cretaceous, Geophys. J. Int., 187(3), 1319-1333, doi:10.1111/j.1365-246X.2011.05245.x.

Creveling, J. R., J. X. Mitrovica, N.-H. Chan, K. Latychev, and I. Matsuyama (2012), Mechanisms for oscillatory true polar wander, Nature, 491(7423), 244-248, doi:10.1038/nature11571.

Daradich, A., J. X. Mitrovica, I. Matsuyama, and J. T. Perron (2008), Equilibrium rotational stability and figure of Mars, Icarus, 194, 463-475. Dziewonski, A. M., and D. L. Anderson (1981), Preliminary Reference Earth Model, Phys. Earth Planet. Inter., 25(4), $297-356$.

Gold, T. (1955), Instability of the Earth's axis of rotation, Nature, 175, 526-529.

Goldreich, P., and A. Toomre (1969), Some remarks on polar wandering, J. Geophys. Res., 74(10), 2555-2567.

Hauck, S. A., and R. J. Phillips (2002), Thermal and crustal evolution of Mars, J. Geophys. Res., 107(E7), 5052, doi:10.1029/2001JE001801.

Lambeck, K., C. Smither, and P. Johnston (1998), Sea-level change, glacial rebound and mantle viscosity for northern Europe, Geophys. J. Int., 134(1), 102-144, doi:10.1046/j.1365-246X.1998.00541.x.

Lefftz, M. (1991), Aspects théoriques de la rotation de la Terre et de son noyau: Influence de la viscosité, PhD thesis, University of Strasbourg.

Matsuyama, I., J. X. Mitrovica, M. Manga, J. T. Perron, and M. A. Richards (2006), Rotational stability of dynamic planets with elastic lithospheres, J. Geophys. Res., 111, E02003, doi:10.1029/2005JE002447.

Mitrovica, J. X. (1996), Haskell [1935] revisited, J. Geophys. Res., 101(B1), 555-569.

Mitrovica, J. X., and A. M. Forte (2004), A new inference of mantle viscosity based upon joint inversion of convection and glacial isostatic adjustment data, Earth Planet. Sci. Lett., 225(1-2), 177-189, doi:10.1016/j.epsl.2004.06.005.

Mitrovica, J. X., and G. A. Milne (1998), Glaciation-induced perturbations in the Earth's rotation: A new appraisal, J. Geophys. Res., 103(E1), 985-1005.

Mitrovica, J. X., J. Wahr, I. Matsuyama, and A. Paulson (2005), The rotational stability of an ice-age Earth, Geophys. J. Int., 161(2), 491-506. Nakada, M., and K. Lambeck (1989), Late Pleistocene and Holocene sea-level change in the Australian region and mantle rheology, Geophys. J. Int., 96, 497-517, doi:10.1111/j.1365-246X.1989.tb06010.x.

Peltier, W. R. (1974), The impulse response of a Maxwell Earth, Rev. Geophys. Space Phys., 12(4), 649-669.

Ricard, Y., G. Spada, and R. Sabadini (1993), Polar wandering of a dynamic Earth, Geophys. J. Int., 113, 284-298, doi:10.1111/j.1365-246X. 1993.tb00888.x.

Richards, M. A., H.-P. Bunge, Y. Ricard, and J. R. Baumgardner (1999), Polar wandering in mantle convection models, Geophys. Res. Lett., 26(12), 1777-1780.

Sabadini, R., and W. R. Peltier (1981), Pleistocene deglaciation and the Earth's rotation: Implications for mantle viscosity, Geophys. J. R. Astron. Soc., 66, 553-578.

Steinberger, B., and R. J. O'Connell (2002), The convective mantle flow signal in rates of true polar wander. Ice Sheets, Sea Level and the Dynamic Earth, Geodynamics Series 29, Copyright 2002 by AGU.

Tsai, V. C., and D. J. Stevenson (2007), Theoretical constraints on true polar wander, J. Geophys. Res., 112, B05415, doi:10.1029/2005JB003923. 
Willemann, R. J. (1984), Reorientation of planets with elastic lithospheres, Icarus, 60, 701-709.

Williams, J.-P., and F. Nimmo (2004), Thermal evolution of the Martian core: Implications for an early dynamo, Geology, 32(2), 97-100, doi:10.1130/G19975.1.

Wu, P., and W. R. Peltier (1984), Pleistocene deglaciation and the Earth's rotation: A new analysis, Geophys. J. R. Astron. Soc., 76, 753-791. Zharkov, V., and T. Gudkova (2005), Construction of Martian interior model, Sol. Syst. Res., 39(5), 343-373, doi:10.1007/s11208-005-0049-7. 